\title{
Foundry-Fabricated Dual-DFB PIC Injection-Locked to Optical Frequency Comb for High-Purity THz Generation
}

\author{
Mu-Chieh Lo ${ }^{1}$, Shi Jia ${ }^{2}$, Deming Kong ${ }^{2}$, Toshio Morioka², Leif K. Oxenløwe², Hao Hu ${ }^{2}$, Guillermo \\ Carpintero ${ }^{1}$ \\ ${ }^{1}$ Universidad Carlos III de Madrid, 28911 Leganés, Madrid, Spain \\ ${ }^{2}$ DTU Fotonik, Technical University of Denmark, DK-2800, Kgs. Lyngby, Denmark \\ Authore-mailaddress: mlo@ing.uc3m.es; huhao@fotonik.dtu.dk; guiller@ing.uc3m.es;
}

\begin{abstract}
We present the first comb injection-locked heterodyne source based on generic foundry-fabricated PIC. The generated $0.4-\mathrm{THz}$ carrier with $\mathrm{Hz}$-level linewidth over 10 -meter wireless link suggests the generic approach is a cost-effective solution for $\mathrm{THz}$ communications. OCIS codes: (060.5625) Radio frequency photonics; (140.3520) Lasers, injection-locked; (250.5300) Photonic integrated circuits.
\end{abstract}

\section{Introduction}

The generation of carrier signals in the microwave, millimeter wave $(\mathrm{mmW}, 30-300 \mathrm{GHz})$ and terahertz $(\mathrm{THz}, 300$ $\mathrm{GHz}-3 \mathrm{THz}$ ) frequency ranges using photonic solutions has been under extensive investigation. It is mainly driven by the advantages that photonics can offer over electronics, particularly the wide tunability of single wavelength laser based on which continuously tunable $\mathrm{mmW} / \mathrm{THz}$ oscillators are simply realized by the beat note between two different laser modes [1]. Developed in the form of photonic integrated circuits (PIC), such mmW/THz synthesizers can provide light weight, small footprint, and low power consumption. Furthermore, in conjugation with the continuous improvement of semiconductor fabrication technologies the open-access InP generic foundry photonic integration approach has emerged that allows active and passive components to be monolithically integrated on the same substrate [2]. Combining two single-wavelength lasers, one high-speed photodiode and one spot-size converter through one $3-\mathrm{dB}$ coupler, the open foundry platform has recently proven the potential for heterodyne $\mathrm{mmW} / \mathrm{THz}$ generation above $1.3 \mathrm{THz}$ at significantly reduced cost. However, the tens-of-MHz laser linewidth and the lack of phase correlation between the two free-running lasers lead to considerable instability of the heterodyne $\mathrm{mmW} / \mathrm{THz}$ signal that limits the practical use [3].

In this paper, we use a coherent optical frequency comb generator (OFCG) based on mode-locked laser (MLL) as a reference to optically injection-lock (OIL) [4] the two DFB laser modes on the foundry-fabricated PIC as shown in Fig. 1. At a wavelength separation of $3.3 \mathrm{~nm}$ in the telecom C-band the two correlated DFB lasers generate a high-spectral-purity $408 \mathrm{GHz}$ carrier with an UTC-PD (uni-traveling-carrier photodiode). Over a 10.7-meter wireless link, the $408-\mathrm{GHz}$ wave is down-converted to $10 \mathrm{GHz}$ at the mixer receiver. The received $10-\mathrm{GHz}$ wave shows a Hz-level linewidth and the single sideband (SSB) phase noise level below $-100 \mathrm{dBc} / \mathrm{Hz}$ at $1-\mathrm{MHz}$ carrier offset is experimentally demonstrated, indicating the potential of foundry approach for $\mathrm{THz}$ communications [5].
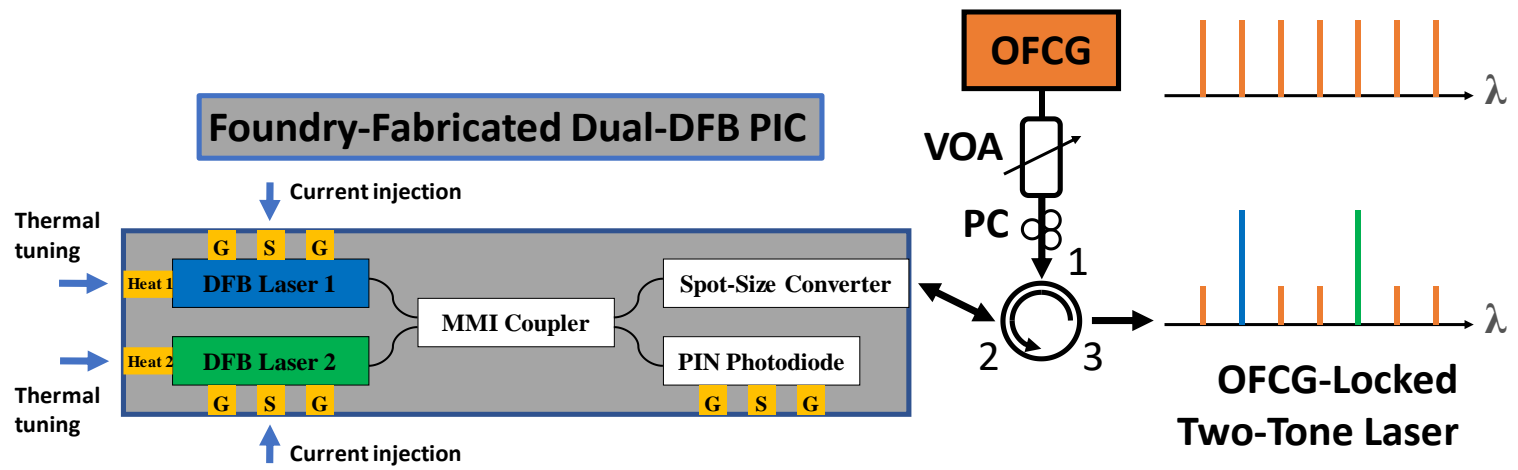

Fig. 1. Schematic of the master-slave optical injection-locking scheme. The OFCG as master injection-locks the two DFB lasers as slave. VOA and PC control and optimize the injection strength and polarization state. The two-tone laser emission locked to the OFCG can be accessed at port 3 of the circulator. The wavelength and intensity of each DFB tone are controlled with the thermal tuning current for integrated heater and injection current. 


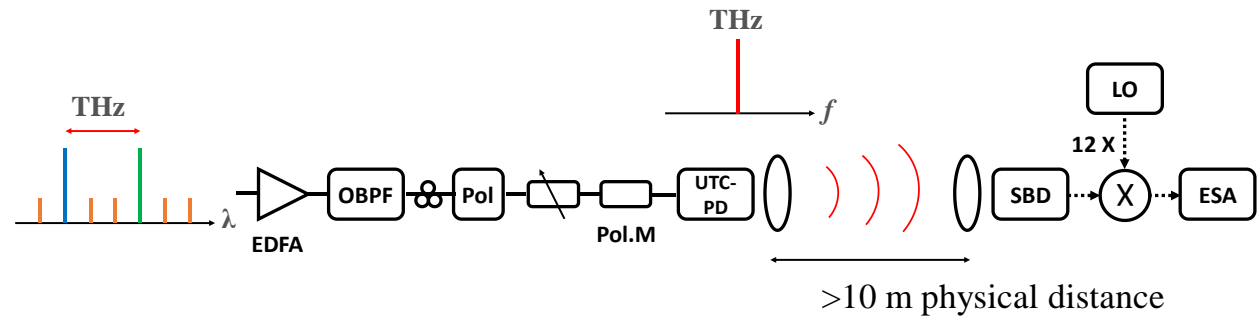

Fig. 2. Experimental setup. The OFCG-locked two-tone laser is amplified, band-pass filtered and optimized in polarization state prior to the UTC-PD. The two tones separated by $408 \mathrm{GHz}$ are heterodyned on the UTC-PD and give rise to a 408-GHz wave. Propagating over 10.7 meters, the received 408-GHz is down-converted on the Schottky Barrier Diode (SBD) mixer with an electrical local oscillator. The collected IF signal is monitored on the ESA.

\section{Experimental Setup}

As shown in Fig.1, on the monolithically integrated dual-DFB PIC the two tunable DFB lasers are coupled to the 3$\mathrm{dB}$ multimode interference (MMI) coupler. The wavelength and intensity of each DFB tone are controlled with the thermal tuning current for integrated heater and injection current. The GSG transmission coplanar waveguide (CPW) supports RF current injection. After the MMI coupler, the superimposed optical two-DFB emission is accessible via the spot-size converter (SSC) on the cleaved facet with anti-reflection (AR) coating. The on-chip PIN-PD allows for on-chip optical heterodyning generation up to $40 \mathrm{GHz}$, far below the THz range. The operation frequency of PINPD and GSG are not within the THz range and thus not studied in this paper. The wavelength spacing between the two free-tuning DFB modes is variable within the range of 0-10.7 nm, corresponding to 0-1.4 THz, in the telecom C-band. To reduce the phase fluctuations between the two free-running tones, an off-the-shelf 9.951-GHz MLLbased OFC is injection-locking the two modes at the same time making them correlated. The two DFB lasers are set to $1555.575 \mathrm{~nm}$ and 1558.975 , locked to two selected modes of the coherent OFC.

The experimental setup for characterizing the OFC-locked two-tone laser is shown in Fig. 2. Before sending the two coherent tones generated by the OFCG-locked dual-DFB PIC into the UTC-PD, an optical bandpass filter (OBPF), a polarization controller (PC), a polarizer, a polarization maintaining variable optical attenuator are is used to control the polarization alignment and optical power of the incoming signal. At the output of the UTC-PD, the $\mathrm{THz}$ signal O/E-converted from the two-tone laser emission with a frequency of $408 \mathrm{GHz}$ is generated and emitted into the $10.7-\mathrm{m}$ wireless link. Two collimating lenses with $100-\mathrm{mm}$ diameter and $200-\mathrm{mm}$ focus length are used at the transmitter and receiver sides. The $408 \mathrm{GHz}$ is then down-converted to the IF of $10 \mathrm{GHz}$ with a SBD mixer, with the 12-time electrical tone being $398 \mathrm{GHz}$ (corresponding to a fundamental rate about $33.17 \mathrm{GHz}$ ). The IF signal is collected with the ESA.

\section{Results and discussion}

The typical behavior of the DFB laser is presented in Fig. 3(a) where the intensity and wavelength continuously increase as the injection current raises. The intensity and wavelength can also be tuned with the heater current. DFB1 covers $1552-1556 \mathrm{~nm}$ and DFB2 covers $1556-1560 \mathrm{~nm}$. In the pure single-wavelength operation, the side-

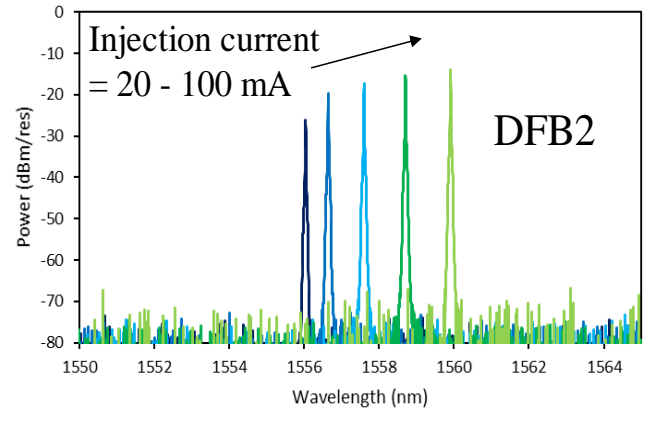

(a)

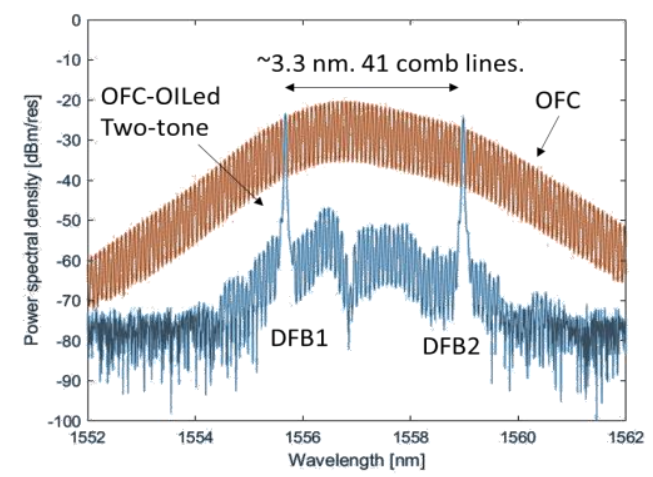

(b)

Fig. 3. (a) The DFB2 laser wavelength shifts from $1556 \mathrm{~nm}$ to $1560 \mathrm{~nm}$ and the intensity increases, as the injection current is varied from $20 \mathrm{~mA}$ to $100 \mathrm{~mA}$. (b) DFB1 and DFB2 are set to $1555.375 \mathrm{~nm}$ and $1558.975 \mathrm{~nm}$, respectively, and optically injection-locked to the MLL-based OFC. Within the $3.3 \mathrm{~nm}(408 \mathrm{GHz})$ wavelength separation 41 comb lines are covered whose comb spacing is $9.951 \mathrm{GHz}$. 


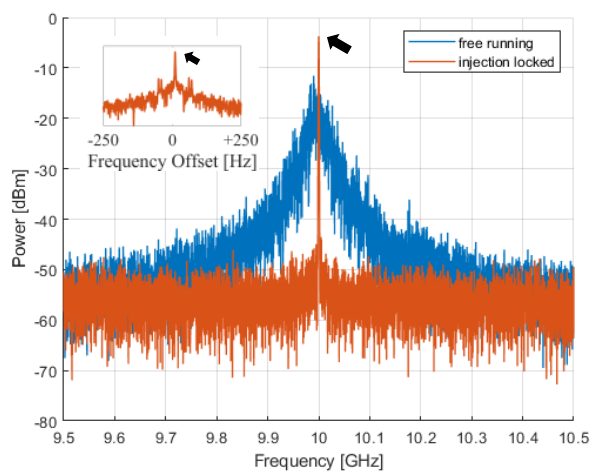

(a)

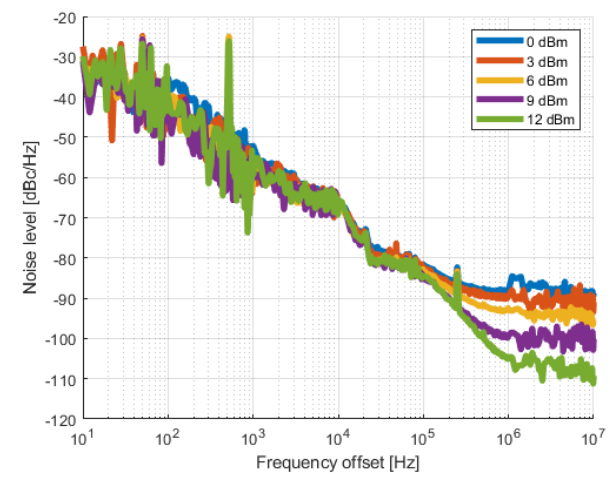

(b)

Fig. 4. (a) The IF collected on the ESA. The blue trace shows the IF signal drifting around $10 \mathrm{GHz}$ when the two DFB lasers are free running, not locked to the OFC. The red trace shows the IF signal at $10 \mathrm{GHz}$ when both DFB lasers are optically injection-locked to the OFC. The locked tone is much narrower and $10 \mathrm{~dB}$ higher than the free-running tone. (b) The single sideband (SSB) phase noise versus different $\mathrm{c} 0 \mathrm{mb}$-injection strengths. With a higher injection strength, the SSB phase noise decreases, particularly in the region where the frequency offset from the carrier is greater than $100 \mathrm{kHz}$.

mode-suppression ratio (SMSR) is around $60 \mathrm{~dB}$. Fig. 3(b) shows the comb spectrum collected at port1 of the optical circulator and the optically injection-locked two-tone emission collected at port3 of the optical circulator. When both laser are aligned to the OFC and properly biased to have the 3.3-nm wavelength separation, the SMSR decreases to $30 \mathrm{~dB}$ due to the unwanted adjacent modes that the comb gives rise to. A comb with wider spacing or a sharp optical filter could improve the SMSR.

The IF electrical spectra are shown in Fig. 4(a). The blue trace shows the down-converted beat-note when the two DFB lasers are not spectrally correlated. The red trace shows the down-converted beat-note when the lasers are properly biased and locked to the OFC, as previously mentioned. The injection strength of the master comb laser is $10 \mathrm{dBm}$, measured with an optical power meter at port1 of the optical circulator. This locked beat-note shows a Hzlevel 3-dB linewidth (see the inset) while the free-running beat-note shows a sub-GHz linewidth. In the free-running operation the two DFB lasers are not correlated and thus generating a huge amount of phase perturbation, leading to the large linewidth as well as the frequency drifting that is related to the long-term frequency stability. The single sideband (SSB) phase noise power spectral density of the down-converted synthesized signal is measured experimentally for the heterodyne between the two DFB lasers separated by $408 \mathrm{GHz}$, as shown in Fig. 4 (b). It demonstrates the effect of injection strength level. In this range a higher injection strength level offers further phase noise reduction further away from the carrier. The phase noise of the heterodyne signal is reduced to the level of less than $-100 \mathrm{dBc} / \mathrm{Hz}$ at $>1 \mathrm{MHz}$ offset when the injection strength is larger than $9 \mathrm{dBm}$.

\section{Conclusions}

We have demonstrated the first comb injection-locked heterodyne $\mathrm{THz}$ generator based on generic foundryfabricated PIC. The characterization results show the improvement in spectral purity and the effect of injection strength level. With such a stabilized and high frequency $(408 \mathrm{GHz})$ carrier, a cost-effective and energy-efficient high-speed THz transmission implementation is expectable using the generic-foundry PIC approach.

\section{Acknowledgements}

The EU H2020 Marie Skłodowska-Curie Grant agreement no. 642355 FiWiN5G, the EU H2020 Marie Sklodowska-Curie grant agreement no. 713683 (COFUNDfellowsDTU), the Danish center of excellence CoE SPOC under Grant DNRF123, and the Villum young investigator program grant of $2 \mathrm{MAC}$.

\section{References}

[1] J. Capmany, and D. Novak, "Microwave photonics combines two worlds," Nature Photonics 1, 319-330 (2007).

[2] M. Smit et al., "An introduction to InP-based generic integration technology," Semiconductor Science and Technology, vol. 29, no. 8, Art. no. 083001 (2014).

[3] M. Lo et al., "Monolithically Integrated Microwave Frequency Synthesizer on InP Generic Foundry Platform," Journal of Lightwave Technology 36, 4626-4632 (2018).

[4] K. Balakier et al, "Optical injection locking of monolithically integrated photonic source for generation of high purity signals above $100 \mathrm{GHz}$," Optics Express 22, 29404-29412 (2014)

[5] T. Nagatsuma et al., "Advances in Terahertz Communications Accelerated by Photonics," Nature Photonics 10, $371-379$ (2016). 